\title{
Hypertelorism-hypospadias- polysyndactyly syndrome
}

INSERM

\section{Source}

INSERM. (1999). Orphanet: an online rare disease and orphan drug data base.

Hypertelorism-hypospadias-polysyndactyly syndrome. ORPHA:2211

Hypertelorism-hypospadias-polysyndactyly syndrome is a very rare syndrome associating an acro-fronto-facio-nasal dysostosis with genitourinary anomalies. 